\title{
Clinicopathological evaluation of intranasal, subcutaneous and intramuscular routes of vaccination against intratracheal challenge of Peste des petits ruminants virus in goats
}

\begin{abstract}
An experiment was conducted to compare the efficacy of intranasal vaccination against caprine Peste des petits ruminant lineage 1 variant virus infection with intramuscular and subcutaneous vaccinations. Twenty four goats were divided into four equal groups. Group 1 was vaccinated intranasally, group 2 was vaccinated subcutaneously, and group 3 intramuscularly, while group 4 was the unvaccinated control. In each group, the vaccinations were carried out once. All goats were challenged intratracheally with PPR virus at a concentration of 106.5 TCID50 two weeks after vaccination and were euthanised 21 days after the challenge. The clinical, lung consolidation, gross and histopathological scores were employed using standard techniques. All data were analysed statistically using the paired ttest and one-way analysis of variance (ANOVA). Group 3 had the highest clinical score following the challenge of vaccinated group while deaths were observed only in group 4. Three goats in group 2 and 3 had pneumonic lung lesions, compared with none in group 1 and all the goats in group 4 . The lung lesions in group 4 were significantly $(\mathrm{P}<0.05)$ severe than in others. Similarly, the lesions in group 2 and 3 were less severe with the right lung more affected $(\mathrm{P}>0.05)$ while group 1 had no consolidation. This study showed that PPR vaccination using subcutaneous, intramuscular and intranasal routes were protective but that of intranasal was found to be easy to apply hence it could be used for the prevention of the disease in small ruminants.
\end{abstract}

Keyword: PPR; Goats; Intranasal vaccination 